\title{
Integer sequences and ellipse chains inside a hyperbola
}

\author{
Hacène Belbachir ${ }^{a}$, László Németh ${ }^{b}$, \\ Soumeya Merwa Tebtoub ${ }^{a}$ \\ ${ }^{a}$ Department of Mathematics, RECITS Laboratory, USTHB, Algiers, Algeria \\ hbelbachir@usthb.dz and hacenebelbachir@gmail.com \\ stebtoub@usthb.dz and tebtoubsoumeya@gmail.com \\ ${ }^{b}$ Institute of Mathematics, University of Sopron, Sopron, Hungary \\ and associate member of RECITS Laboratory, USTHB \\ nemeth.laszlo@uni-sopron.hu \\ Submitted: May 12, 2020 \\ Accepted: June 24, 2020 \\ Published online: June 30, 2020
}

\begin{abstract}
We propose an extension to the work of Lucca [Giovanni Lucca, Integer sequences and circle chains inside a hyperbola, Forum Geometricorum, Volume 19. 2019, 11-16]. Our goal is to examine chains of ellipses inside a hyperbola, and we derive recurrence relations of centers and minor (major) axes of the ellipse chains. We also determine conditions for these recurrence sequences to consist of integer numbers.
\end{abstract}

Keywords: Ellipse chains, circle chains, hyperbola, integer sequences.

MSC: 52C26, $11 \mathrm{~B} 37$.

\section{Introduction}

Let us consider the hyperbola $\mathcal{H}$ with the canonical equation

$$
\frac{x^{2}}{a^{2}}-\frac{y^{2}}{b^{2}}=1,
$$


and foci $( \pm c, 0)$, where $a$ and $b$ are positive real numbers and $c^{2}=a^{2}+b^{2}$. Lucca [1] examined a tangential chain of circles inside the branch $x>0$ of the hyperbola so that the $i$-th circle with center $\left(x_{i}, 0\right)$ and radius $r_{i}$ is tangent to the hyperbola and to the preceding and succeeding circles labelled by indexes $i-1$ and $i+1$, respectively. He showed that in case of certain ratios $\frac{b}{a}$ the sequences $\left\{\frac{x_{i}}{x_{0}}\right\}_{i=0}^{\infty}$ and $\left\{\frac{r_{i}}{r_{0}}\right\}_{i=0}^{\infty}$ are integers.

In our article, we extend Lucca's work. We define and examine a special chains of ellipses inside the branch $x>0$ of the hyperbola, when the ratio of the minor and major axis is fixed. It is a natural extension of Lucca's circle chains. We describe the recurrence relations of sequences of centers, major and minor axes, which determine another type of proof to give integer sequences. Therefore, we are able to provide more integer sequences than in case of Lucca's circle chains.

\section{Ellipse chains inside a branch of hyperbola}

Let us define a chain of ellipses with the following properties:

- The center of each ellipse lies on the $x$-axis, inside the branch $x>0$ of the hyperbola (1.1), the semi-axes are parallel to the coordinate lines. More precisely, the canonical equation of the $i$-th ellipse centered at point $\left(u_{i}, 0\right)$ $\left(u_{i}>0\right)$ is

$$
\frac{\left(x-u_{i}\right)^{2}}{\alpha_{i}^{2}}+\frac{y^{2}}{\beta_{i}^{2}}=1,
$$

where $2 \alpha_{i}>0$ is the width and $2 \beta_{i}>0$ is the height of the ellipse (Figure 1 ). If $\alpha_{i}>\beta_{i}$, then the focal axis of the $i$-th ellipse is coincident with the $x$-axis, if $\alpha_{i}<\beta_{i}$, then it is parallel to the ordinate axis, and if $\alpha_{i}=\beta_{i}$, then the $i$-th ellipse is a circle. In the figures $\alpha_{i}<\beta_{i}$.

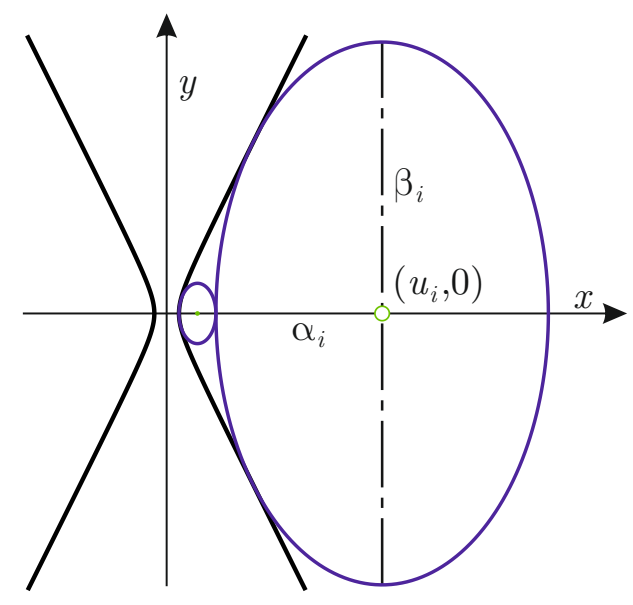

Figure 1: An ellipse chain inside a hyperbola 
- The ellipses (2.1) are tangent to the hyperbola (1.1).

- The first ellipse (Figure 2) is tangent (and do not intersect at any points) to the hyperbola at its vertex $A$ having coordinates $(a, 0)$, so:

$$
u_{0}=a+\alpha_{0}
$$

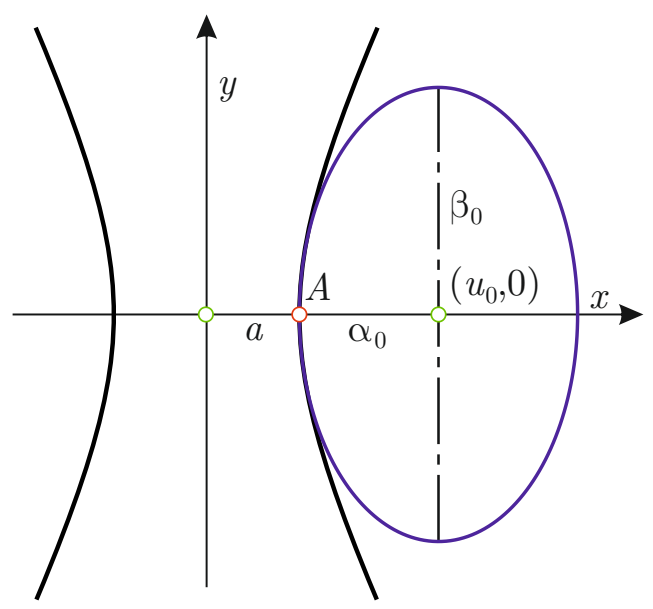

Figure 2: First ellipse of a chain

- The curvature in case of the hyperbola must not be bigger than the curvature in case of the first ellipse at $A$, otherwise they are not only tangent to each other at $A$, but also the ellipse intersects the hyperbola at two other points. Thus,

$$
\frac{\alpha_{0} b^{2}}{a} \geq \beta_{0}^{2}
$$

where $\frac{a}{b^{2}}$ and $\frac{\alpha_{0}}{\beta_{0}^{2}}$ are, respectively, the curvatures of the hyperbola and the first ellipse at point $A$.

- In order that the first ellipse provides the best touching to the hyperbola at $A$, we have to require the same curvature of the ellipse and the hyperbola at $A$. That is why we restrict inequality (2.2) to equation

$$
\frac{\alpha_{0} b^{2}}{a}=\beta_{0}^{2} .
$$

- The ellipses are mutually tangent. It means that the $i$-th ellipse is tangent to the $(i-1)$-th ellipse and to the $(i+1)$-th ellipse, so we have

$$
u_{i}-u_{i-1}=\alpha_{i}+\alpha_{i-1}
$$


- We pose $\frac{\beta_{i}}{\alpha_{i}}=m$, where $m=\frac{\beta_{0}}{\alpha_{0}}=\frac{\sqrt{\frac{\alpha_{0} b^{2}}{a}}}{\alpha_{0}}=\frac{b}{\sqrt{\alpha_{0} a}}$. Hence the ellipses are similar to each other.

In order to achieve our goal, we consider the system of equations

$$
\left\{\begin{array}{l}
\frac{x^{2}}{a^{2}}-\frac{y^{2}}{b^{2}}=1 \\
\frac{\left(x-u_{i}\right)^{2}}{\alpha_{i}^{2}}+\frac{y^{2}}{\beta_{i}^{2}}=1
\end{array}\right.
$$

Now we give the general relations between $u_{i}$ and $\alpha_{i}$. From (2.4) we have

$$
\left(\frac{\beta_{i}^{2}}{\alpha_{i}^{2}}+\frac{b^{2}}{a^{2}}\right) x^{2}-2 \frac{\beta_{i}^{2}}{\alpha_{i}^{2}} u_{i} x+\frac{\beta_{i}^{2}}{\alpha_{i}^{2}} u_{i}^{2}=b^{2}+\beta_{i}^{2},
$$

due to the tangency condition the discriminant $\Delta$ of $(2.5)$ must be zero so:

$$
\frac{\Delta}{4}=\frac{\beta_{i}^{4}}{\alpha_{i}^{4}} u_{i}^{2}-\left(\frac{\beta_{i}^{2}}{\alpha_{i}^{2}}+\frac{b^{2}}{a^{2}}\right)\left(\frac{\beta_{i}^{2}}{\alpha_{i}^{2}} u_{i}^{2}-b^{2}-\beta_{i}^{2}\right)=0 .
$$

Then we have

$$
u_{i}^{2}=\left(\frac{a^{2}}{b^{2}}+\frac{\alpha_{i}^{2}}{\beta_{i}^{2}}\right)\left(b^{2}+\beta_{i}^{2}\right)
$$

Since $\frac{\beta_{i}}{\alpha_{i}}=m$, then

$$
u_{i}^{2}=\left(\frac{a^{2}}{b^{2}}+\frac{1}{m^{2}}\right)\left(b^{2}+m^{2} \alpha_{i}^{2}\right)
$$

and

$$
u_{i-1}^{2}=\left(\frac{a^{2}}{b^{2}}+\frac{1}{m^{2}}\right)\left(b^{2}+m^{2} \alpha_{i-1}^{2}\right) .
$$

By subtracting (2.7) from (2.6) and by remembering (2.3), we obtain

$$
\left\{\begin{array}{l}
u_{i}+u_{i-1}=m^{2}\left(\frac{a^{2}}{b^{2}}+\frac{1}{m^{2}}\right)\left(\alpha_{i}-\alpha_{i-1}\right), \\
u_{i}-u_{i-1}=\alpha_{i}+\alpha_{i-1}
\end{array}\right.
$$

Since $m^{2}=\frac{b^{2}}{a \alpha_{0}}$, we gain

$$
\left\{\begin{array}{l}
u_{i}+u_{i-1}=\left(1+\frac{a}{\alpha_{0}}\right)\left(\alpha_{i}-\alpha_{i-1}\right), \\
u_{i}-u_{i-1}=\alpha_{i}+\alpha_{i-1}
\end{array}\right.
$$


In order to give the expression of $u_{i}$ and $\alpha_{i}$, we have to solve the system (2.8), after some algebraical steps we obtain

$$
\begin{gathered}
u_{i}=\left(2 \frac{\alpha_{0}}{a}+1\right) u_{i-1}+2\left(1+\frac{\alpha_{0}}{a}\right) \alpha_{i-1}, \\
\alpha_{i}=2 \frac{\alpha_{0}}{a} u_{i-1}+\left(2 \frac{\alpha_{0}}{a}+1\right) \alpha_{i-1} .
\end{gathered}
$$

Since $\beta_{i}=m \alpha_{i}$, we have

$$
\beta_{i}=\frac{b}{a \sqrt{\alpha_{0} a}}\left(2 \alpha_{0} u_{i-1}+\left(2 \alpha_{0}+a\right) \alpha_{i-1}\right) .
$$

We should notice that the case $m=1$ (the ellipses are circles) provides $\alpha_{0}=\beta_{0}=$ $\frac{b^{2}}{a}$, so we are in [1].

We can represent the expression of $u_{i}$ and $\alpha_{i}$ in matrix form as follows:

$$
\left(\begin{array}{c}
u_{i} \\
\alpha_{i}
\end{array}\right)=\left(\begin{array}{cc}
2 \frac{\alpha_{0}}{a}+1 & 2\left(1+\frac{\alpha_{0}}{a}\right) \\
2 \frac{\alpha_{0}}{a} & 2 \frac{\alpha_{0}}{a}+1
\end{array}\right)\left(\begin{array}{c}
u_{i-1} \\
\alpha_{i-1}
\end{array}\right) .
$$

\subsection{Sequences}

In this paragraph, we give recurrence relations of centers and minor (major) axes of the ellipse chains.

Theorem 2.1. The sequences $\left\{u_{i}\right\},\left\{\alpha_{i}\right\}$ and $\left\{\beta_{i}\right\}$ are the same second-order linear homogeneous recurrence sequences

$$
\ell_{i}=2\left(2 \frac{\alpha_{0}}{a}+1\right) \ell_{i-1}-\ell_{i-2} \quad(i \geq 2),
$$

with initial values $\alpha_{0} \in \mathbb{R}^{+}, \beta_{0}=\frac{b \alpha_{0}}{\sqrt{a \alpha_{0}}}, u_{0}=a+\alpha_{0}, \alpha_{1}=\frac{\alpha_{0}}{a}\left(3 a+4 \alpha_{0}\right), \beta_{1}=$ $\frac{b \sqrt{\alpha_{0}}\left(3 a+4 \alpha_{0}\right)}{a^{3 / 2}}$ and $u_{1}=\frac{a^{2}+5 \alpha_{0} a+4 \alpha_{0}^{2}}{a}$.

Proof. From the sum of (2.8) we have

$$
2 u_{i}=\left(2+\frac{a}{\alpha_{0}}\right) \alpha_{i}-\frac{a}{\alpha_{0}} \alpha_{i-1}
$$

and

$$
2 u_{i-1}=\left(2+\frac{a}{\alpha_{0}}\right) \alpha_{i-1}-\frac{a}{\alpha_{0}} \alpha_{i-2} .
$$

The sum of them combined again by (2.8) after some calculation yields

$$
\alpha_{i}=2\left(2 \frac{\alpha_{0}}{a}+1\right) \alpha_{i-1}-\alpha_{i-2}
$$

Similarly, $u_{i}=2\left(2 \frac{\alpha_{0}}{a}+1\right) u_{i-1}-u_{i-2}$. Moreover, since $\beta_{i}=m \alpha_{i}$, the recurrence is true for $\beta_{i}$. The initial values come from the equations (2.9)-(2.11). 
Let $\bar{u}_{i}=\frac{u_{i}}{u_{0}}, \bar{\alpha}_{i}=\frac{\alpha_{i}}{\alpha_{0}}$, and $\bar{\beta}_{i}=\frac{\beta_{i}}{\beta_{0}}$. Then we obtain the following theorem as a corollary of Theorem 2.1 .

Theorem 2.2. The sequences $\left\{\bar{u}_{i}\right\},\left\{\bar{\alpha}_{i}\right\}$ and $\left\{\bar{\beta}_{n}\right\}$ are second-order linear homogeneous recurrence sequences (2.12) with initial values $\bar{u}_{0}=\bar{\alpha}_{0}=\bar{\beta}_{0}=1$, $\bar{u}_{1}=1+\frac{4 \alpha_{0}}{a}, \bar{\alpha}_{1}=\bar{\beta}_{1}=3+\frac{4 \alpha_{0}}{a}$.

Corollary 2.3. The sequences $\left\{\bar{\alpha}_{i}\right\}$ and $\left\{\bar{\beta}_{i}\right\}$ are the same.

Circle chains defined by Lucca [1] are the special cases $\alpha_{i}=\beta_{i}=r_{i}$ of our chains. The following corollary gives the recurrence relation for this unique case.

Corollary 2.4. If $\alpha_{0}=\frac{b^{2}}{a}$, then the sequences $\left\{\bar{u}_{n}\right\},\left\{\bar{\alpha}_{n}\right\}$ are second-order linear homogeneous recurrence sequences

$$
\ell_{i}=2\left(2 \frac{b^{2}}{a^{2}}+1\right) \ell_{i-1}-\ell_{i-2} \quad(i \geq 2),
$$

with initial values $\bar{u}_{0}=\bar{\alpha}_{0}=1, \bar{u}_{1}=1+\frac{4 b^{2}}{a^{2}}, \bar{\alpha}_{1}=3+\frac{4 b^{2}}{a^{2}}$.

\subsection{Integer sequences}

In this paragraph, we determine conditions to relate the ellipse chains with integer sequences.

Theorem 2.5. In case of any positive integer $k$, if

$$
\alpha_{0}=k \frac{a}{4},
$$

then the sequences $\left\{\bar{u}_{i}\right\}_{i \in \mathbb{N}}$ and $\left\{\bar{\alpha}_{i}\right\}_{i \in \mathbb{N}}$ are integer sequences, and their recurrences are

$$
\ell_{i}=(k+2) \ell_{i-1}-\ell_{i-2} \quad(i \geq 2),
$$

with initial values $\bar{u}_{0}=\bar{\alpha}_{0}=1, \bar{u}_{1}=1+k, \bar{\alpha}_{1}=3+k$.

Proof. Theorem 2.2 shows, that the first two items of the sequences are integers if $k=\frac{4 \alpha_{0}}{a}$ is integer. Then the first coefficient of recurrence relation $(2.12)$ is $k+2$, which guarantees that all the other items of the sequences are integers.

Example 2.6. We give now some examples of integer sequences that can be obtained for different values of $k$.

- For $k=1$;

The sequence $\{\bar{\alpha}\}=\{1,4,11,29,76,199, \ldots\}$ which corresponds to the bisection of Lucas sequence is classified in The On-Line Encyclopedia of Integer Sequences (OEIS [2]) as $A 002878$.

- For $k=2$;

The sequence $\{\bar{u}\}=\{1,3,11,41,153,571,2131, \ldots\}$ is classified in OEIS as A001835. 
- For $k=3$;

The sequence $\{\bar{u}\}=\{1,4,19,91,436,2089, \ldots\}$ appears in OEIS as $A 004253$. The sequence $\{\bar{\alpha}\}=\{1,6,29,139,666,3191, \ldots\}$ which corresponds to the Chebyshev even index $U$-polynomials evaluated at $\frac{\sqrt{7}}{2}$ is classified in OEIS as $A 030221$.

- For $k=4$;

The sequence $\{\bar{\alpha}\}=\{1,7,41,239,1393,8119, \ldots\}$ which corresponds to the Newman, Shanks-Williams (NSW) numbers is classified in OEIS as A002315.

In case of Lucca's circle chains for integer sequences when $\alpha_{0}=\frac{k a}{4}=\frac{b^{2}}{a}$ we obtain the following corollary.

Corollary 2.7. If $r_{i}=\alpha_{i}=\beta_{i}$ and the ratio $\frac{b}{a}$ is given by

$$
\frac{\sqrt{k}}{2}=\frac{b}{a}, \quad k=1,2, \ldots
$$

then the sequences $\left\{\bar{u}_{i}\right\},\left\{\bar{r}_{i}\right\}$ are integer sequences. Their recurrences are

$$
\ell_{n}=(k+4) \ell_{n-1}-\ell_{n-2} \quad(n \geq 2),
$$

with initial values $\bar{u}_{0}=\bar{\alpha}_{0}=1, \bar{u}_{1}=1+k, \bar{\alpha}_{1}=3+k$.

Comparing Corollary 2.7 and Lucca's similar main theorem, we find that Corollary 2.7 contains more integer sequences. Only if $k$ is a square number, then the sequence appears in Lucca's theorem. We also mention that for relatively small $k$ a huge number of integer sequences are arising in OEIS [2].

Acknowledgements. For H. B. and S. M. T. this work was supported by the grant of DGRSDT, number C0656701. For L. N. this work has been made in the frame of the "EFOP-3.6.1-16-2016-00018 - Improving the role of the research + development + innovation in the higher education through institutional developments assisting intelligent specialization in Sopron and Szombathely".

We would like to thank the anonymous referee for carefully reading the manuscript and for his/her useful suggestions and improvements.

\section{References}

[1] G. LuCCA: Integer sequences and circle chains inside a hyperbola, Forum Geometricorum 19 (2019), pp. 11-16.

[2] N. J. A. Slonne: The On-Line Encyclopedia of Integer Sequences, https://oeis.org. 